\title{
Using an integrated monitoring platform to communicate geotechnical risk to project stakeholders
}

\author{
WJ Conrad Canary Systems, Inc., USA
}

AM Neuwirt Canary Systems, Inc., USA

\begin{abstract}
Geotechnical data is frequently analysed by highly qualified engineers, but often a challenge is relaying what the data means to other project stakeholders for them to make informed decisions. From equipment operators, to corporate leaders, to the general public, it is an engineer's responsibility to be able to communicate in an effective manner the meaningful information about risk that is applicable to each group/person. As datasets grow larger, monitoring technology advances, and the input from stakeholders becomes increasingly important. Integrated monitoring platforms must allow engineers to interpret technical information into an understandable form for logical decision-making as it relates to design, safety, environmental management, operations, and regulations. Summarising key metrics, filtering out noise, and aggregating data types for a simplified view of a project are all necessary to effectively allow an engineer to communicate risk and uncertainty with a broad audience. This paper will examine how integrated monitoring platforms assist in bridging the transferring of information about risk to the different types of technical and non-technical stakeholders involved in geotechnical projects; specifically in mining operations.
\end{abstract}

Keywords: monitoring, stakeholders, risk communication

\section{$1 \quad$ Introduction}

Risk management is often a complex topic as it applies to mining. Mining companies must consider economic and societal impacts, in the form of environmental loss, operational shutdown, safety, and decreased public perception, to their investors, employees, and the public (Pot et al. 2015). Many factors, such as commodity prices, information security, safety, and changes in regulations impact each operation. One risk that should be valued by all stakeholders in mining operations is the continual geotechnical risk that must be managed. From the beginning of operations, all the way through a property's closure phase, stakeholders are affected by the geotechnical risk associated with mining activities. This risk is often mitigated by implementing a continuous monitoring program that is used by a team of experienced geotechnical engineers.

According to Siebenmann et al. (2015), the rationale in geotechnical engineering for monitoring the health of a structure is to provide a warning system to prevent a critical failure, as well as to reduce uncertainty that underlies all geotechnical work. Also important while monitoring a site's conditions, is to be able to inform all stakeholders in a project so they can make adequate decisions as they relate to their responsibilities or interests. For these groups to make informed decisions, each requires specific information about geotechnical risk (Leung et al. 2008).

The number of these stakeholders is continually growing as individuals see an increasing value in the geotechnical aspects of an operation due to higher uncertainty in markets and other operational risks. Stakeholder groups commonly influenced by geotechnical risk include:

- Operations personnel.

- Geotechnical technicians.

- Mine planners.

- Corporate geotechnical groups. 
- Geotechnical engineering consultants.

- Site and corporate management.

- Regulatory bodies.

- Shareholders and the general public.

As groups become more involved, the decision-making process has an increasing amount of value, and new demands are placed on geotechnical engineers to translate their knowledge and analysis into relatable information (Pot et al. 2015). As mine sites evolve, risk information should be continually translated in a comprehensible form to all stakeholders of geotechnical data, both technical and non-technical. Consideration of how geotechnical risk will be relayed to stakeholders must be taken as early as the risk assessment phase, as "the filtering of information about hazards... may profoundly alter the form and context of the risk information produced and conveyed" (Kasperson et al. 1988).

Datasets are constantly increasing due to the often hundreds of sensors and data sources deployed at each mine. This has led to integrated monitoring platforms becoming an important tool in the field of geotechnical engineering to assist in risk management and communicate this risk to others. These systems are largely used to make in-house decisions that are relayed to operations and upper management about the conditions in the field that affect safety and production. Geotechnical monitoring systems come in many forms, whether they are application or web-based, and have a variety of tools and features, such as custom configurations, alarming and notification capabilities, and data presentation methods, that relate to the data analysis needs of engineers tasked with assessing geotechnical risks. The goal of the tools is to allow engineers to fully understand the conditions that the site faces to improve the safety of those working in the field and reduce the financial risk related to geotechnical risk/hazards of the operation. In understanding the geotechnical environment, an engineer can effectively communicate this risk to other stakeholders for them to be able to make their own informed decisions.

\section{$2 \quad$ Fundamental parts of integrated monitoring systems}

\subsection{Integration capabilities}

For a monitoring system to be effective in managing and presenting geotechnical risk, the platform must allow all sources of data on a site to be displayed in one platform. This is accomplished by offering a variety of tools to allow connection to loggers in the field and other databases, as well as allowing the input of manually collected data. Connections to other systems include the development of application programming interfaces (APIs), custom scripts, or file imports.

An integrated platform is important to be able to determine any correlations within different types of monitoring, instead of trying to make decisions based off discrete, independent datasets. Many types of sensors are installed on sites to provide a redundancy check and make up for another type of sensor's weaknesses. For example, ground-based radar systems have known line-of-sight issues, and prisms and GPS units have atmospheric errors. When the data from these different sensors are integrated into a single interface, geotechnical engineers can overcome the shortfalls of each and have the opportunity to correlate data from different sources for the determination of an area's stability.

Another aspect of an integrated monitoring platform is that it allows geotechnical engineers to have to learn only one interface for working with data. This provides simplicity in an already hectic work environment. Technologies and software are also continuously improved upon over time, so working with one platform reduces the amount of continual education required by the engineers, allowing them to have more time to focus on analysing and relaying conditions to other stakeholders.

The ability for determining different user levels and geotechnical data access is also an important part of relaying information to various stakeholders. One system can be used to manage all users and all data sources. By giving partial or full access to only read, or both read and edit, the system can be configured by 
way of maps, charts, reports, and alarms to relay pertinent information to specific stakeholder groups. This can be performed manually or automatically.

\subsection{Platform configurations}

The determination of how calculations, charts, reports, and alarms are configured within an integrated monitoring platform is the most significant and challenging part of a system. Geotechnical teams must set up the best way to transform the raw data by using prior experience and best industry practice. If done incorrectly, outputs from the system will mislead stakeholders on the conditions of a site. Consistency within a monitoring system must also be maintained to provide a simpler analysis platform for the geotechnical team and to allow the communication of results to others to be uniform.

Decisions must be made as far as what calculations are relevant to each data type, what time periods should be used for rates of change, the alarm limits associated with site characteristics, and who should be notified at each level of an alarm for the best course of action and remediation. Enhanced features, such as using trendlines, allow systems to be able to alarm from predicted values to provide a proactive approach to risk management, instead of the typical reactive approach widely used in the current geotechnical profession. Trending allows stakeholders to better understand how risks relate to the future and can assist in more accurately made decisions than simply analysing and understanding historical data. Figure 1 displays a trendline example from the three-dimensional inverse velocity of a prism placed on a slope failure. Other examples of trendlines include using them for predicting hydrologic response when piezometric data is correlated with rainfall predictions, as well as changes in stress if a correlation with drawpoint extraction is determined in a block cave mine. Differing reports must also be able to be configured to adequately relay information to various stakeholders. Site management will not be concerned with instrument reliabilities in the same manner as geotechnical technicians tasked with ensuring a fully operational system.

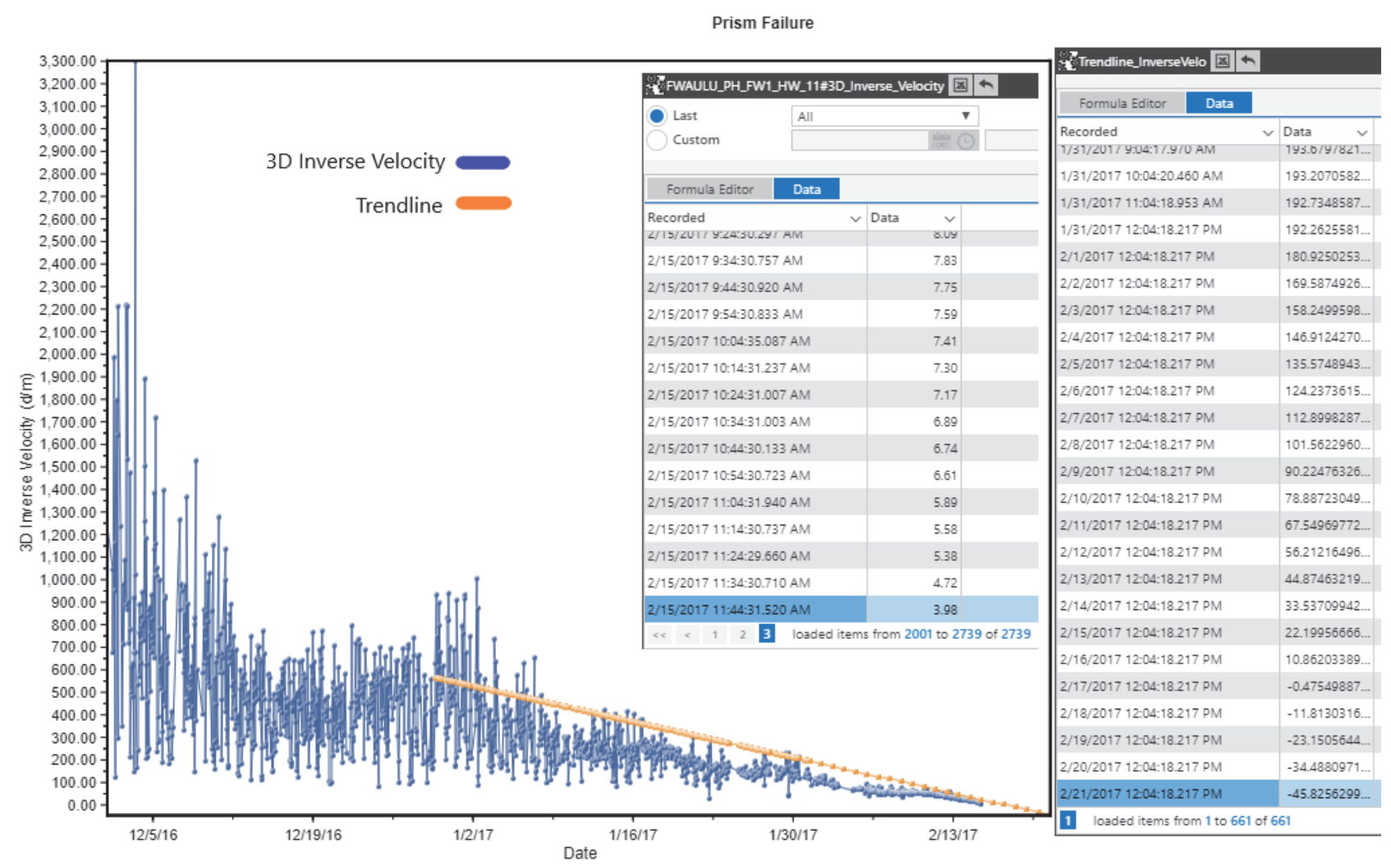

Figure 1 Trendline creation using three-dimensional inverse velocity prism data. Specific time periods within the dataset are used for determining the line. Users can set the trendline type, as well as extents and interval of prediction into the future 
A method of logging and acknowledging events that occur within a monitoring system is vital as well to ensure to various stakeholders that an adequate overview of the geotechnical aspects of a site are being performed. Management groups, both at the site and corporate level, as well as regulatory bodies, must have a means of oversight to ensure that standards are being met and issues are being appropriately responded to for reducing the inherent risk of mining.

\subsection{Data presentation}

Data presentation in geotechnical monitoring systems allows for the simplification of the meaning for differing types of data consumers. Presentations are combined through the software interface and printed outputs. Typically, the best method of transferring information across varying levels of knowledge is by transferring data into useful images to assist visualisation. Image windows, which display georeferenced maps, relay instrument locations and status to allow the visualisation of how an area is behaving. Contouring and scalable vectors give visual indicators of the data without comparing the values themselves. Filtering tools accessible within an image window also assists different stakeholders in removing unnecessary information that is not pertinent to their needs, as shown in Figure 2. Charting tools plot calculated data that allow trends to be identified, and reports can be created to focus on what a stakeholder views according to their relation to the monitoring system and geotechnical conditions. Depending on the stakeholder, data tables may also be useful in relaying the data in a meaningful way.

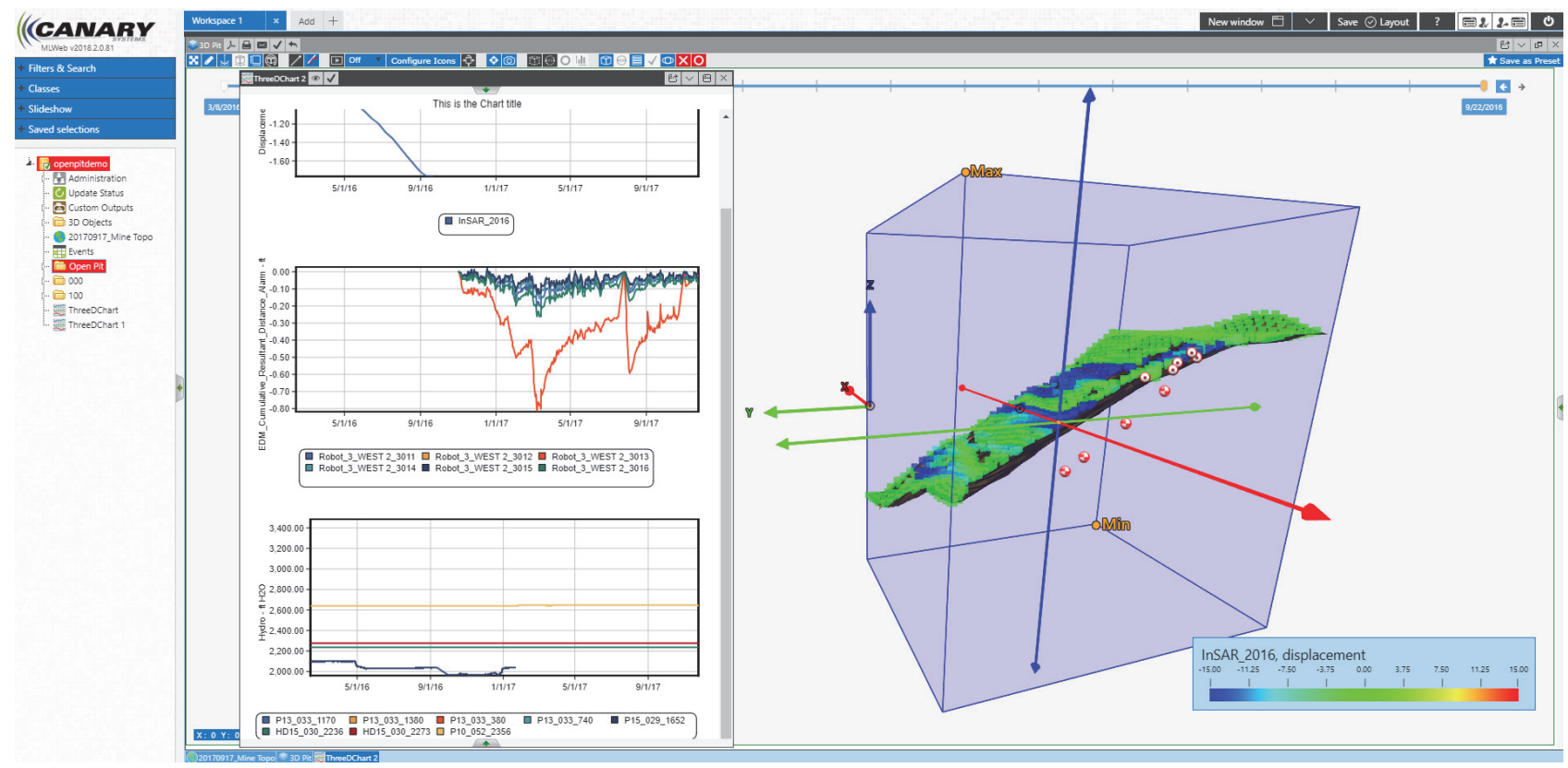

Figure 2 Integrated 3D scene with an active viewing filter of InSAR, prisms, and piezometers

\section{Communicating with different stakeholders}

\subsection{Operations}

The relay of information between geotechnical engineers and the site operations team is critical for ensuring that miners are working in safe areas and not exposed to hazardous ground conditions. Information such as the location of higher-risk operating areas, and what conditions are expected and should be carefully observed, is vital in keeping employees safe. Mining rates may even need to be changed as hazards arise. Ideally, engineers should be communicating with operations personnel on a shift-by-shift basis for keeping them informed on the most current conditions when risk levels affect personnel safety.

Geotechnical monitoring platforms assist in this transfer of information by providing the key metrics as to how areas are performing. Preconfigured calculations, charts, and reports that automatically update allow 
for an analysis of the data with little user input after setup. Simple reports should be created for an operator's specific area of work, use familiar colour coding (e.g. green, yellow, and red) for hazard levels, contain a map of hazard areas, and relay a few numeric values, such as 24-hour prism movement or microseismic events over the last day to best give individuals a frame of reference. An example status report for an underground section is found in Figure 3. These reports may be posted in changing rooms, reviewed at safety meetings, and/or available on monitors posted inside equipment.

Underground Geotechnical Microseimic Daily Monitoring Report

July 09, 2018

\begin{tabular}{ccc}
\multicolumn{3}{c}{ Minimum and Maximum Magnitudes } \\
\hline Zone & Minimum & Maximum \\
\hline $\mathbf{1}$ & -1.025 & -0.389 \\
$\mathbf{2}$ & -0.949 & -0.083 \\
$\mathbf{3}$ & -0.780 & 0.067
\end{tabular}

\begin{tabular}{|c|c|c|c|c|c|}
\hline \multirow{2}{*}{ Re-Entry Protocol } & \multicolumn{2}{|c|}{ Additional Working Area Criteria } & \multirow{2}{*}{ Zone 1 } & \multirow{2}{*}{ Zone 2 } & \multirow{2}{*}{ Zone 3 } \\
\cline { 2 - 3 } & Poisson Distribution & Energy Index & & \multirow{2}{*}{ OPEN } \\
\hline GREEN & GREEN & GREEN & \multirow{2}{*}{ OPEN } & OPEN & \multirow{2}{*}{ OPEN } \\
\hline GREEN & GREEN & GREEN & GREEN & & \\
\hline GREEN & GREEN & GREN &
\end{tabular}

\begin{tabular}{|c|c|c|}
\hline \multirow{2}{*}{ Criteria } & \multicolumn{2}{|c|}{ Comments } \\
\hline & GREEN/OPEN & RED/CLOSED \\
\hline Re-Entry Protocol & $\begin{array}{l}\text { Moment Magnitude }<=0.7 \\
\text { Less than } 3 \text { events with Moment Magnitude }<=-0.3\end{array}$ & $\begin{array}{c}\text { Moment Magnitude }>=0.7 \\
\text { More than } 3 \text { events with Moment Magnitude }>=-0.3\end{array}$ \\
\hline Poisson Distribution & Less than the threshold of 10 events & More than the threshold of 10 events \\
\hline Energy Index & Incremental EI $<=1$ & Incremental EI >= 1 \\
\hline Open/Closed & All of the criterias are 'GREEN' & One or more criterias are 'RED' \\
\hline
\end{tabular}

Figure 3 Reporting example of an active underground section displaying the status of mining zones relating to the section's microseismicity

In addition to distributed reports, alarms and notifications placed on the results of calculations enable the system to alert individuals without them having to log into the system. Operations personnel should be added to these notifications to reduce the notification time of hazardous conditions. These notifications must be well-identified as what the response should be for the alert, whether to heighten awareness or in worse case scenarios, evacuate, and can be in direct relation to trigger action response plans (TARPs) that have been implemented. Once a responsible individual receives a notification about an abnormal situation, this individual may log into the system to validate the alarm, and if confirmed, continue with the plan of response for that issue. As with any automated system, continual analysis of the reliability of the data and limits should be performed to ensure that false alarms are avoided.

Not only can current data levels be used in alarms, but predicted levels may be as well using identified correlations in conjunction with trendline functions. For example, rainfall amounts could be determined to have a correlation with highwall displacement. By using weather forecasting, the predicted rainfall could indicate a potential slope stability issue that can be mitigated before the rain occurs. Combining these correlations with trendline functions gives operations more time to respond to geotechnical issues and possibly avoid them altogether. 


\subsection{Geotechnical teams/personnel}

Geotechnical teams are extremely vital to the health of an operation's geotechnical monitoring system. They are often the ones tasked with going out into the field to set up monitoring equipment and instrumentation, and for troubleshooting, making observations, and performing field measurements. Instrument and equipment reliability, system parameters such as battery voltage, and the locations of instruments or measurement locations should be communicated to these individuals for them to appropriately maintain a system.

Monitoring platforms must be able to configure data tolerances and provide reliability data in an accessible form for personnel to consume. To reduce the amount of manual labour in sorting through data, instrument reports with configurable fields should be created within the system, as shown in Figure 4. Validity percent, last valid date, last valid reading, maximum readings, and minimum readings over a defined timeframe relay the information needed to identify which data sources are having problems. Instrument reports may be scheduled to be sent automatically from the system on a daily, weekly, or monthly basis to ensure personnel are being continually notified of issues. Data update notifications also allow teams to be alerted when instruments haven't reported any valid data for a specified time. To assist with identifying specific locations to visit, georeferenced images with icons representing an instrument or monitoring point are best used.

\begin{tabular}{llllll} 
& \multicolumn{5}{c}{$\begin{array}{c}\text { Open Pit } \\
\text { Battery Voltage Reliability Report }\end{array}$} \\
Name & Valid Percent & \multicolumn{2}{c}{$\begin{array}{c}\text { Balid Reading Date } \\
\text { Valid Reading Value }\end{array}$} & $\begin{array}{l}\text { Maximum } \\
\text { Minimum }\end{array}$ \\
P10_046_\#P10_046_BV & 100 & $4 / 30 / 2018$ & 12.96 & 14.23 & 12.70 \\
P10_051_\#P10_051_BV & 100 & $4 / 30 / 2018$ & 12.80 & 14.25 & 12.58 \\
P10_052_\#P10_052_BV & 100 & $4 / 30 / 2018$ & 13.02 & 14.23 & 12.75 \\
P10_070_\#P10_070_BV & 100 & $4 / 30 / 2018$ & 12.98 & 14.37 & 12.75 \\
P10_077_\#P10_077_BV & 100 & $4 / 30 / 2018$ & 12.93 & 14.59 & 12.70 \\
P10_080_\#P10_080_BV & 100 & $4 / 30 / 2018$ & 12.97 & 14.28 & 12.74 \\
P11_029_\#P11_029_BV & 100 & $4 / 30 / 2018$ & 12.86 & 14.26 & 12.56 \\
P11_035_\#P11_035_BV & 100 & $4 / 30 / 2018$ & 12.92 & 14.25 & 12.68
\end{tabular}

Figure 4 Battery voltage reliability report, output by geotechnical software, used by geotechnical technicians to identify monitoring stations with battery issues

It is also important for geotechnical technicians to understand the risk management value of the monitoring system to ensure that any issues within the system are taken care of in a timely manner and accurate data is being reported. This is often best achieved by immersing them in the process of how data is used to assist other stakeholders, and the value that the collected geotechnical data provides these groups. Training geotechnical technicians to contribute in the configuration of the monitoring system for inputting instrument-specific properties and setting up outputs within the system, assists with their familiarity of the monitoring program, as well as reduces the workload for engineers who can focus on data analysis.

\subsection{Mine planners}

The relationship between the geotechnical team on a site and mine planners is crucial to the overall success of an operation, and bridges the gap between assumed results and actual results. Data gathered during exploration programs give an insight into the rock types and geologic structures that will be encountered as mining progresses, but there are still many unknowns on how the rock will behave once excavated. Design parameters and/or geometry, such as mining rates or drawpoint sequencing, may need to be altered if plans are found to either be unsafe or, alternatively, overdesigned. Sometimes, hazardous areas may need to be avoided entirely. Mine planners benefit from understanding how mining rates and locations are affecting the stability of a slope/excavation. Summary reports that relay hazardous areas identified by the monitoring analysis and mitigation recommendations for these areas are most helpful for these stakeholders.

\subsection{Corporate geotechnical groups}

The main purpose of a corporate geotechnical group, as it relates to monitoring, is to ensure that the site is exercising due diligence in implementing recommended data sources, keeping a system healthy, understanding the challenges of their property, and knowing how to react to any issues encountered. This is 
accomplished by corporate groups through continual check-ins, monitoring system audits, and training programs. In turn, site engineers are tasked with communicating their shortfalls in knowledge about the meaning of their data, as well as keeping an open mind as to the geotechnical challenges that they are encountering.

Corporate geotechnical engineers need to be provided a higher level of information, such as in the form of summary reports, than the site engineers, and direct access to the monitoring system to gather additional details about specific areas to ensure compliance to standards. These individuals are key in the spreading of knowledge as it pertains to calculations, charting, reporting, and alarming configurations.

\subsection{Geotechnical engineering consultants}

External geotechnical consultants are often tasked with the design of the mine itself, from determining highwall angles, to defining optimal dimensions of underground workings. Consultants initially start with the same dataset as mine planners, which is gathered from an exploration program and used in modelling software. Over time, due to an implemented monitoring system, knowledge about a site significantly grows, and models can be continuously updated with the most recent information gathered by a monitoring system. Continually feeding geotechnical data back to design and modelling software assists in creating the safest, most economic mine plan by comparing predicted verses actual results and revising for the best plan.

Geotechnical monitoring systems act as an intermediary in the communication from geotechnical engineers to the consultant stakeholder. Raw data is gathered and processed, and can then be passed on in the form of comma separated value (CSV) files or through an API for consumption into other software to update models. Continual feedback from modelling results can then be used to update assumed parameters in calculations and alarm conditions configured in the geotechnical monitoring software, as shown in Figure 5. Since geotechnical data directly influences modelling, consultants should also have direct read access to the database to ensure that the data that they are inputting into their models is accurate and trustworthy.

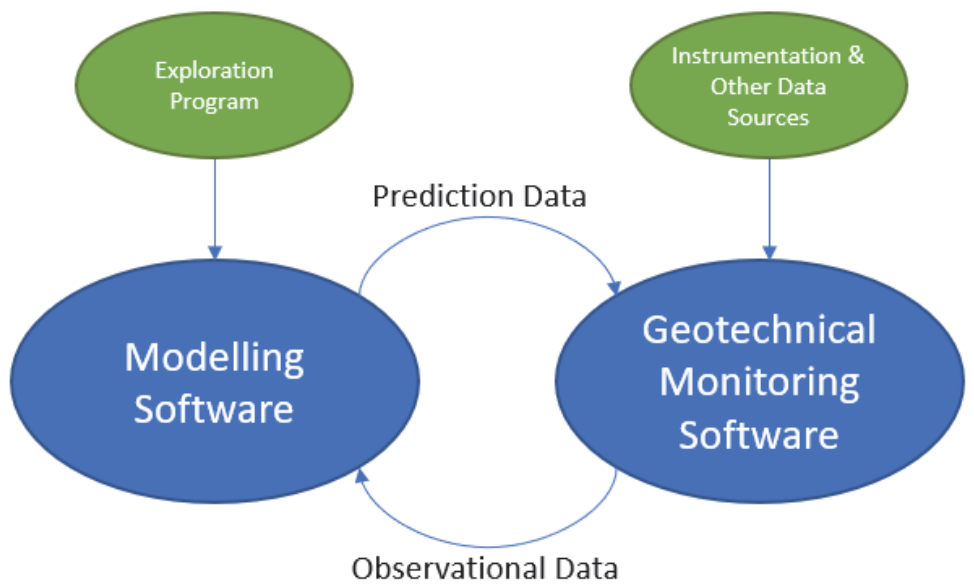

Figure 5 Cyclic nature of dataflow between modelling and geotechnical monitoring software

\subsection{Site and corporate management}

Relaying geotechnical risk information to those in site and corporate management roles is often a difficult task. A typical geotechnical engineer is not accustomed to translating data and probabilities into financial risk, and upper management at a mine site or at the corporate level may not be well-informed on the technical aspects that affect the mining process. Due to the site and corporate management stakeholder groups needing to ensure that the mine is economic as well as safe, engineers must be able to relate what they have analysed from their monitoring system to key performance indicators (KPIs) to provide a quick, understandable method of relaying risk. Such KPIs include probabilities of failure and the remaining life of an area's stability. The size and locations of hazardous or potentially hazardous areas should also be relayed to 
management to be factored into the overall risk of the operation. Charts and reports created in monitoring platforms can effectively relay this information.

\subsection{Regulatory bodies}

Regulatory bodies are primarily concerned with compliance to a country's laws and regulations, and ensuring that sites are diligent in providing a safe working atmosphere for their employees. Historically, geotechnical engineers have had to compile large datasets and present them in a specified format, specific to the agency requesting the analysis. With today's monitoring systems, these reports can be imitated directly in the platforms themselves, and automatically updated with data from a selected timeframe. This reduces the overall time spent on these reports and provides regulatory bodies consistency in the communication of the data's analysis and presentation of results. Figure 6 displays a page of a governmental report created in a custom reporting tool that is automatically produced by a deployed system every six months.
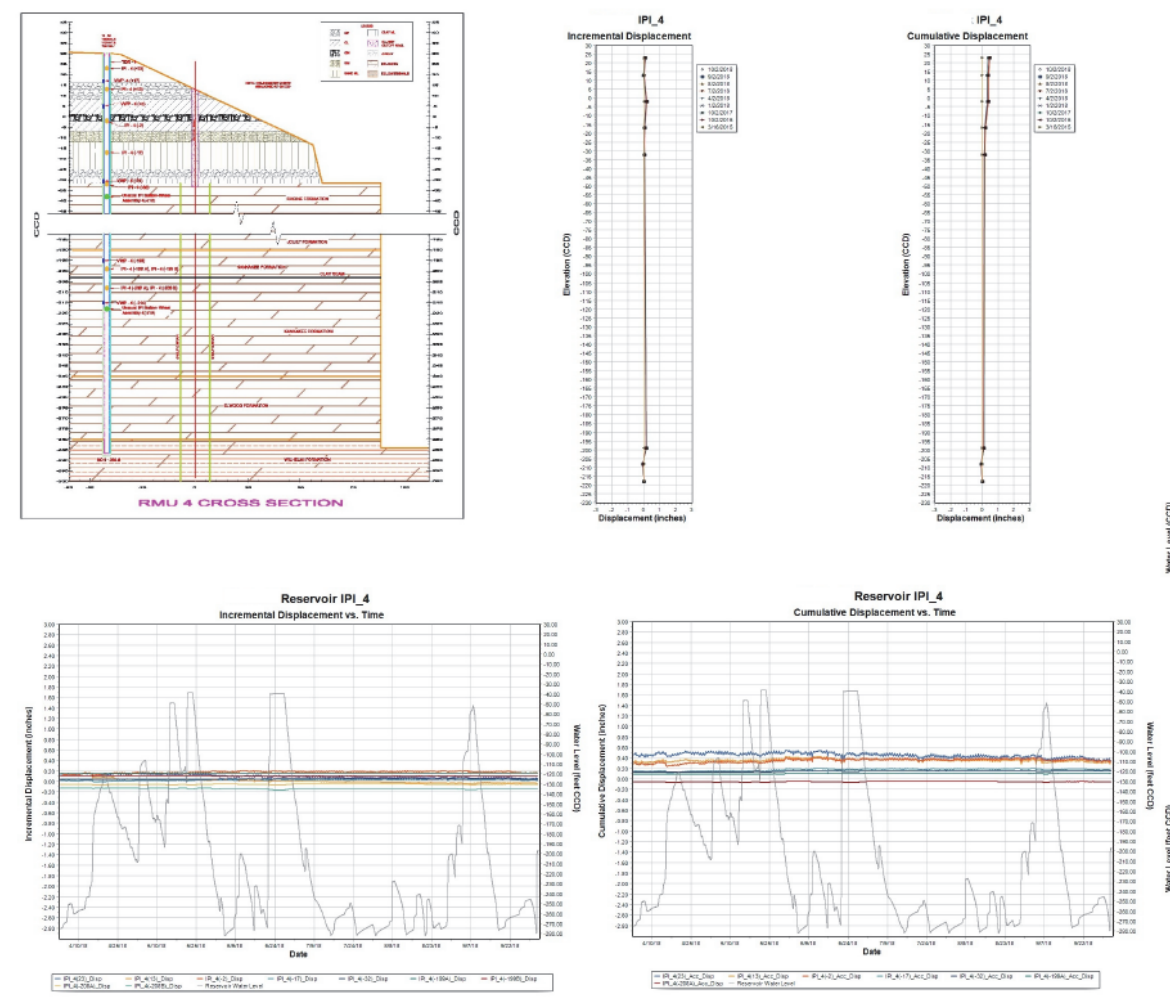
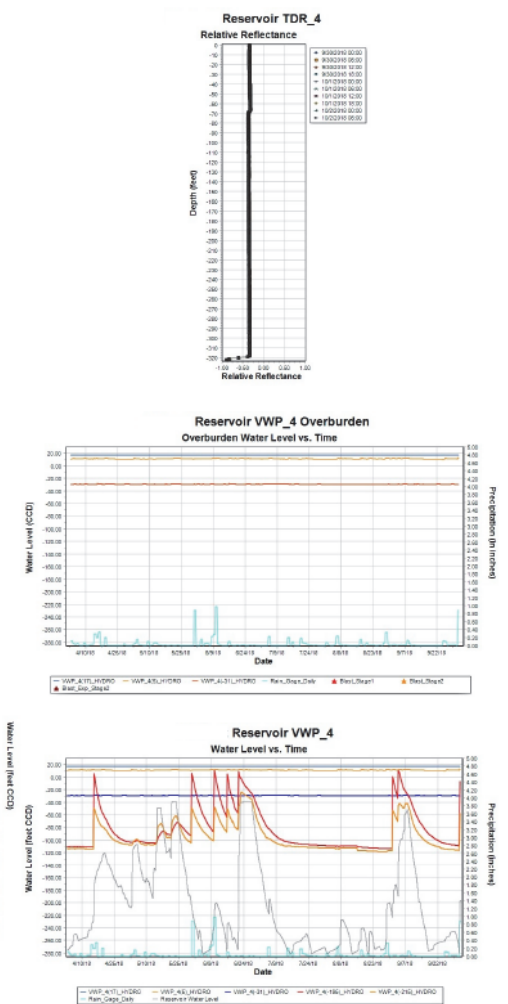

Figure 6 Custom report created for governmental submission, automatically produced by the system every six months

\subsection{Shareholders and general public}

The perception of a mine site's risk in the public eye is often conceived from prior events that have made media headlines. Even small risks, or events associated with risk, often cause a strong response from the public and result in significant impacts, both economically and societally (Kasperson et al. 1988). Many individuals not in the mining or geotechnical industry do not realise the breadth that many mining operations take to ensure that mines are stable, and risks are being managed through geotechnical monitoring systems. Shareholders of mining companies are more likely to have this knowledge, but still can shy away from investing if the general public does not have the trust of a particular company or industry. This group of individuals is directly impacted by any negative or positive risk, whether perceived, or actually true.

Geotechnical monitoring systems can be a tool to relay that sites are being proactive and good stewardships to their investors and the surrounding communities. By allowing the public to see the results of monitoring, and thus open the risks an operation faces to the surrounding community, it pushes those in design and system management roles to provide a more well-designed, safer environment. This also permits the public 
to trust an operation and experience themselves that which operators are telling them is true. With web-based platforms that many current monitoring platforms are designed around, the public can access a glimpse of the system with up-to-date data and information. Annual summary reports can also be created in platforms for public and shareholder consumption as a continual update on how effectively risks are being managed. As with any release of data, the parameters associated with the data and its meaning must be effectively communicated. Percentages and aggregations with associated alarm values are methods of presenting the information in a more concise form to assist personnel in relaying this information. Instead of sharing actual geotechnical data, operators may alternatively share system parameter statistics, such as the number and type of instruments actively monitoring a property, to relay to the public that any geotechnical risk is being actively managed.

\section{$4 \quad$ Summary}

In summary, the responsibilities of geotechnical engineers to communicate risk to such a broad audience is extremely complicated. Many different stakeholders, both technical and non-technical, rely on their expertise to be able to make decisions regarding their own responsibilities, and must be communicated with in a dissimilar manner. Monitoring systems can assist geotechnical engineers in navigating this difficult terrain by providing a single, integrated platform for managing large amounts of data from extremely different data sources. Tools for mapping, charting, and reporting within monitoring platforms enable complex data to be analysed and communicated efficiently by summarising key metrics, filtering out noise, and aggregating data types. In turn, risk is managed more properly, and mine sites are able to operate safer and more economically.

\section{References}

Kasperson, RE, Renn, O, Slovic, P, Brown, HS, Emel, J, Goble, R, Kasperson, JX \& Ratick, S 1988, 'The social amplification of risk: a conceptual framework', Risk Analysis, vol. 8, no. 2, pp. 177-187, https://doi.org/10.1111/j.1539-6924.1988.tb01168.x

Leung, SW, Mak, S \& Lee, BLP 2008, 'Using a real-time integrated communication system to monitor the progress and quality of construction works', Automation in Construction, vol. 17, no. 6, pp. 749-757, https://doi.org/10.1016/j.autcon.2008.02.003

Pot, R, Roelse, FP, van Der Meer, MT, Nushi, BRI, \& Nelemans, JP 2015, 'The observational method in a real-time, multi- stakeholder environment', in AFT Schweckendiek \& D van Tol (eds), Proceedings of the 5th International Symposium on Geotechnical Safety and Risk: Geotechnical Risk and Safety V, IOS Press, Amsterdam, pp. 990-996, http://doi.org/10.3233/978-1-61499580-7-990

Siebenmann, R, Yu, HT \& Bachus, R 2015, 'UCIMS: advances in geotechnical construction and performance monitoring', Journal of Rock Mechanics and Geotechnical Engineering, vol. 7, no. 2, pp. 207-212, https://doi.org/10.1016/j.jrmge.2015.03.002 
